\title{
RELAÇÕES ENTRE INDICADORES DE DESEMPENHO: UM ESTUDO EXPLORATÓRIO EM EMPRESAS LOCALIZADAS EM SERRA TALHADA/PE ${ }^{1}$
}

\author{
RELATIONSHIP BETWEEN PERFORMANCE INDICATORS: AN EXPLORATORY \\ STUDY AMONG COMPANIES LOCATED IN SERRA TALHADA/PE
}

\author{
Antônio André Cunha Callado ${ }^{2}$ \\ Aldo Leonardo Cunha Callado ${ }^{3}$ \\ Luciano Pires de Andrade ${ }^{4}$
}

\begin{abstract}
RESUMO: Este artigo teve o objetivo de identificar a estrutura e padrões de relações existentes entre os indicadores de desempenho utilizados em empresas localizadas no município de Serra Talhada/PE. Para a realização dessa pesquisa foram analisadas 49 empresas e investigados 17 indicadores de desempenho. $\mathrm{O}$ instrumento utilizado para a coleta de dados foi o questionário. Para atingir o objetivo proposto, foram utilizadas três abordagens metodológicas multivariadas complementares: a análise de agrupamentos, o escalonamento multidimensional e a análise fatorial. Os resultados obtidos demonstraram que apenas nove indicadores de desempenho puderam ser considerados como pertencentes a algum agrupamento de maneira significativa. Através da análise de agrupamentos foi observada a presença de três agrupamentos. O primeiro agrupamento foi composto pelos indicadores de desempenho que tratam da qualidade dos produtos, da fidelização dos clientes, do volume de vendas e dos investimentos em modernização. O segundo agrupamento foi formado pelas variáveis que representam a participação dos produtos no faturamento e pela capacidade operacional. E o terceiro agrupamento foi composto pela rentabilidade, pelo valor do patrimônio e pelo valor da empresa. Esses resultados foram confirmados pelo escalonamento multidimensional e pela análise fatorial.
\end{abstract}

PALAVRAS-CHAVE: Medição de desempenho. Indicadores de desempenho. Abordagem multivariada.

ABSTRACT: This article aims to identify the structure and patterns of relationships between the performance indicators used in companies located in Sierra Talhada/PE. To achieve this objective, 49 companies were analyzed and 17 performance indicators were investigated. The instrument used for data collection was a questionnaire. Three multivariate complementary methodological approaches were used: the clusters' analysis, the multidimensional scaling and factor analysis. The results showed that only nine performance indicators could be regarded as belonging to any group in a significant manner. Through the analysis of groups was the presence of three groups. The first group was composed of the performance indicators that deal with product quality, the loyalty of customers, sales volume and investment in modernization. The second group was formed by the variables representing the participation of the products in the billing and operational capacity. And the third group was composed of profitability, the value of assets and the value of the company. These results were confirmed by both multidimensional scaling and factor analysis.

KEYWORDS: Measurement of performance. Performance indicators. Multivariate approach.

\footnotetext{
${ }^{1}$ Artigo Recebido em 21.01.2009. Revisado por pares em 03.07.2009. Recomendado em 20.07.2009 por Leomar dos Santos (Editor Adjunto). Publicado em 03.09.2009.

Organização Responsável pelo periódico: Universidade regional de Blumenau - FURB - www.furb.br/rn
}

\footnotetext{
${ }^{2}$ Universidade Federal Rural de Pernambuco - andrecallado@yahoo.com.br

${ }^{3}$ Universidade Federal da Paraíba- aldocallado@yahoo.com.br

${ }^{4}$ Universidade Federal Rural de Pernambuco -
} 


\section{INTRODUÇÃO}

$\mathrm{Na}$ gestão estratégica empresarial, as informações sobre seu desempenho, sejam em seu ambiente interno ou externo, norteiam sua tomada de decisão visando à eficácia empresarial e seu posicionamento competitivo no mercado. Além disso, as informações direcionam as mudanças e aprimoram as previsões sobre o futuro.

Gonçalves (2002) ressalta que todas as empresas, de um modo geral, necessitam de um sistema de avaliação de desempenho, uma vez que a realização contínua do processo de avaliação permite que a empresa conheça a eficiência e a eficácia de suas ações, bem como o comportamento das pessoas, os processos e os programas da organização.

O processo de seleção do elenco de indicadores de desempenho apropriados para auxiliar os gestores de uma determinada empresa deve estar baseado em etapas seqüenciadas de maneira lógica. Os indicadores escolhidos devem estar orientados para o futuro, buscando dar contorno aos objetivos estabelecidos, bem como traduzir as metas de médio e longo prazo.

Nesta perspectiva, MacArthur (1996) conceitua indicador de desempenho como sendo a quantificação de como estão sendo realizadas as atividades com o propósito de comparar com as metas especificadas.

Sobre o papel dos indicadores de desempenho no processo gerencial das organizações, Martins (2004) afirma que este processo de gestão de desempenho é o meio pelo qual uma organização administra seu desempenho de acordo com as estratégias corporativas e funcionais, bem como seus objetivos.

Sink e Tutlle (1989) consideram que o uso eficaz de sistemas de mensuração de desempenho pode ser dificultado pelos paradigmas adotados pelos gestores. Por outro lado, Araújo (2001) ressalta que a necessidade de inter-relação entre os elementos de eficácia organizacional, fatores críticos de sucesso e medidas de desempenho tem sido amplamente reconhecidas, mas adverte que a definição das medidas de desempenho pode ser influenciada pela cultura da empresa e pelas condições mercadológicas dentre outros.

Ao discutir a eficiência e a eficácia de uma organização, o termo desempenho incorpora um significado universal que representa uma avaliação e que pode assumir um relevante papel no processo de elaboração de estratégias de longo prazo, bem como analisar sua situação dentro da indústria na qual ela esteja inserida.

Os indicadores de desempenho são elementos fundamentais para a mensuração de performance, bem como na definição das variáveis que melhor representem o desempenho geral de uma empresa. Para cada setor de atividades, podem ser elaborados grupos distintos de indicadores de desempenho, obedecendo as próprias características específicas, assim como da assimilação de conceitos metodológicos pelos profissionais responsáveis por sua elaboração.

O objetivo deste trabalho é identificar a estrutura e padrões de relações existentes entre os indicadores de desempenho utilizados em empresas localizadas no município de Serra Talhada/PE.

\section{INDICADORES DE DESEMPENHO}

Uma apropriada definição para o termo indicador de desempenho é condição primordial para entender seu significado institucional, bem visualizar a amplitude de sua utilização. Neely et al. (1996) definem o indicador de desempenho como sendo um meio utilizado para quantificar a eficiência e/ou a eficácia de uma tomada de decisão feita pela empresa. Um indicador de desempenho, portanto, permite às organizações saberem o que estão fazendo e quais os resultados de suas ações. 
Zilber e Fischmann (2002) ressaltam que os indicadores de desempenho permitem verificar a propriedade com que as decisões foram tomadas e, eventualmente, corrigir e readequar o processo vigente de gestão. Portanto, um indicador de desempenho possibilita que uma determinada organização possa saber a evolução dos efetivos resultados decorrentes de suas atividades enquanto unidade de negócio.

A correta definição sobre um indicador de desempenho consiste no primeiro passo para o entendimento de seu significado institucional, bem como a visualização da amplitude de sua aplicação.

Kaplan e Norton (1992) consideram que nenhum indicador de desempenho isolado será capaz de prover uma clara representação da performance de uma organização, nem poderá focar todas as áreas relevantes ao mesmo tempo.

Brignall (1997) considera que as medidas de avaliação de desempenho podem ser contextualizadas em duas dimensões distintas, que são:

- Resultado do sucesso competitivo $\Rightarrow$ Através de medidas de competitividade e medidas financeiras;

- Determinantes do sucesso competitivo $\Rightarrow$ Através de medidas de qualidade, de flexibilidade, de utilização de recursos e de inovação.

Os indicadores de desempenho podem ser classificados em financeiros e nãofinanceiros. Para Morissete (1977), indicador financeiro é uma medida quantitativa, expressa em valor monetário, resultante das ações tomadas pelas empresas. Como exemplos de indicadores financeiros têm-se: lucro e retorno sobre os investimentos. E o indicador nãofinanceiro é uma medida quantitativa que não será expressa em valor monetário, ou seja, o indicador não-financeiro é apresentado em uma métrica diferente de uma unidade monetária. Como exemplos de indicadores não-financeiros têm-se: participação no mercado e evolução do volume de produção.

Kaplan e Norton (1997) argumentam que os indicadores financeiros são inadequados para orientar e avaliar a trajetória da empresa em um ambiente competitivo, pois são indicadores de ocorrência, contam parte, mas não toda a história das ações passadas, bem como não fornece orientações adequadas para as ações que devem ser realizadas hoje e amanhã para criar valor financeiro futuro.

Banker, Potter e Srinivasan (2000) apresentam que as medidas não-financeiras são melhores indicadores de medidas de desempenho futuro que as medidas financeiras, auxiliando os administradores a focarem suas ações em perspectivas de longo prazo.

Verificando-se a qualidade da performance através da utilização dos indicadores de desempenho, os gestores podem tomar decisões mais seguras sobre as estratégias da organização, uma vez que possuem informações específicas para fins gerenciais.

Segundo Macedo e Silva (2004), os métodos de classificação que consideram os aspectos financeiros e não financeiros tendem a assumir uma importância especial, visto que o desempenho acaba por ser afetado por variáveis de ambas naturezas.

Walter, Bornia e Kliemann Neto (2000) afirmam que um dos grandes desafios da gestão empresarial contemporânea é a consideração de valores não-financeiros na avaliação do desempenho.

A utilização de indicadores como referência quantitativa de mensuração de desempenho não implica necessariamente que haja um sistema integrado de mensuração de performance. Wouters e Sportel (2004) afirmam que os gestores utilizam diferentes formas de indicadores de desempenho, mas, freqüentemente, não os contextualizam no âmbito de um sistema de mensuração de desempenho. Estes autores ainda ressaltam a necessidade de aprofundar as investigações empíricas sobre a contribuição dos indicadores de desempenho como instrumento facilitador da gestão. 
Outro aspecto relevante está associado ao caráter dinâmico do ambiente competitivo que precisa ser considerado quando da definição do elenco de indicadores relevantes de desempenho, bem como dos procedimentos operacionais inerentes ao sistema de mensuração de desempenho.

Sobre a sintonia necessária entre os indicadores de desempenho e o contexto competitivo das empresas, Nadjmi, Rigas e Fan (2005) afirmam que a performance de um negócio é dinâmica e pode sofrer alterações ao longo do tempo e que estas mudanças precisam ser consideradas para que os devidos ajustes possam ser implementados no sistema de mensuração de desempenho.

Os indicadores de mensuração de desempenho têm recebido uma atenção significativa nos últimos dez anos, tanto por parte dos acadêmicos, através de suas investigações empíricas e proposições de frameworks, quanto por parte dos gestores através da utilização de indicadores de desempenho como parâmetro de avaliação organizacional e fazem parte da agenda de pesquisa para os próximos anos.

Sobre a inserção das investigações sobre indicadores de desempenho, Neely et al. (2005) destaca as seguintes tendências: a) Desenvolver e implementar uma gestão de performance ao invés de sistemas de mensuração; b) Mensurar a performance de cadeias de suprimento e redes de empresas ao invés de se restringir ao âmbito interno das organizações; c) Mensurar ativos tangíveis e intangíveis, bem como a gestão interna; d) Desenvolver sistemas dinâmicos de mensuração de desempenho; e) Dar flexibilidade aos sistemas de mensuração de desempenho para que eles possam se ajustar às mudanças.

\section{PROCEDIMENTOS METODOLÓGICOS}

Dentro da perspectiva abordada neste trabalho faz-se necessário caracterizar o foco metodológico que será adotado para atingir o objetivo proposto. Esta pesquisa possui uma natureza exploratória.

Gil (1999) afirma que as pesquisas exploratórias são importantes, pois elas têm a finalidade de desenvolver, esclarecer e modificar conceitos e idéias, bem como formular problemas mais precisos ou hipóteses pesquisáveis em estudos posteriores.

Nesta mesma vertente, Andrade (1999) considera que uma pesquisa exploratória visa agregar informações sobre um determinado assunto com a finalidade de definir objetivos, formular hipóteses ou descobrir novos enfoques a serem investigados.

Comentando sobre as características das pesquisas exploratórias, Gil (2002) destaca que o planejamento deste tipo de pesquisa é bastante flexível e deve considerar diversas perspectivas sobre o fato a ser estudado.

Sobre as pesquisas exploratórias, Vergara (2003) considera que, dada sua natureza, elas não comportam hipóteses iniciais, mas que podem surgir ao longo da realização da pesquisa.

\subsection{Universo e Amostra investigada}

De acordo com Leite (1978), com a definição do universo delimita-se o campo de pesquisa em termos temporais, geográficos, setoriais ou qualquer outra dimensão cabível com base na disponibilidade ou obtenibilidade de dados ou com o fundamento nos objetivos e nos custos da execução da pesquisa.

Para acessar e obter informações sobre o universo a ser investigado, Silver (2000) propõe a utilização de listas especializadas como fonte de consulta. A fonte utilizada na presente pesquisa foi o cadastro do Clube dos Diretores Lojistas (CDL) da cidade de Serra 
Talhada/PE. O universo desta pesquisa foi composto pelas 167 empresas listadas no cadastro do CDL. A amostra investigada nesta pesquisa foi composta por 46 empresas.

\subsection{Indicadores de desempenho investigados}

Diante do objetivo proposto nesta pesquisa, foram investigados os seguintes indicadores de desempenho:

- Participação dos produtos no faturamento;

- Rentabilidade do patrimônio líquido;

- Valor do patrimônio líquido;

- Valor da empresa;

- Qualidade do produto;

- Fidelização dos clientes;

- Volume de vendas;

- Investimento em propaganda;

- Investimento em modernização;

- Capacidade operacional;

- Evolução do número de clientes;

- Tempo de atendimento ao cliente;

- Rotatividade de empregados;

- Número de reclamações;

- Tempo para solução de reclamações;

- Evolução do número de funcionários;

- Investimento em treinamento.

\subsection{Procedimentos da coleta de dados}

A presente pesquisa possui aspectos exploratórios, que envolve entrevistas com pessoas que tiveram ou têm experiência práticas com o problema pesquisado. Para a Coleta de dados desta pesquisa foi utilizada a técnica da entrevista estruturada.

De acordo com Chizzotti (1991), entrevista estruturada é um tipo de comunicação entre um pesquisador que pretende coletar informações sobre fenômenos e indivíduos que detenham essas informações e possam emiti-las.

Para Gil (2002), a entrevista estruturada é quando se desenvolve a partir de uma relação fixa de perguntas. Esta técnica consiste em fazer uma série de perguntas a um informante, conforme roteiro preestabelecido, onde esse roteiro pode constituir-se de um formulário/questionário que será aplicado da mesma forma a todo os informantes/sujeitos da pesquisa, para que se obtenham respostas para as mesmas perguntas.

$\mathrm{O}$ instrumento utilizado para a coleta de dados foi o questionário. Sobre o uso de questionário, Chizzotti (1991) indica que ele é um conjunto de questões pré-elaboradas, sistemática e seqüencialmente dispostas em itens que constituem o tema da pesquisa, com o objetivo de suscitar dos informantes respostas por escrito ou verbalmente sobre o assunto que os informantes saibam opinar.

Nesta mesma perspectiva, Gil (2002) afirma que a elaboração de um questionário consiste basicamente em traduzir os objetivos específicos da pesquisa em itens bem redigidos.

Outros aspectos decisivos para a escolha desse instrumento de coleta de dados foram as vantagens apresentadas pela sua utilização, que segundo Richardson (1999) são as 
seguintes: as respostas e perguntas fechadas são fáceis de codificar; o entrevistado não precisa escrever e por último, as perguntas fechadas facilitam o preenchimento total do questionário.

\subsection{Método de Análise}

Para a identificação da estrutura e padrões de relações existentes entre os diversos indicadores de mensuração de desempenho investigados, esta pesquisa considerou uma abordagem multivariada.

Análise multivariada é definida por Hair et al. (2005) como sendo o conjunto de métodos estatísticos que simultaneamente analisam múltiplas medidas sobre cada um dos indivíduos ou objetos sob investigação.

De acordo com Hair et al. (2005) e Rodrigues e Paulo (2007), o tipo de relação investigada (relação de dependência ou de interdependência) é um fator central para a definição da abordagem multivariada apropriada. A análise de relações de dependência pode ser realizada através das seguintes técnicas: modelagem de equações simultâneas, análise de relações canônicas, análise multivariada de variância, regressão múltipla e análise discriminante. Por outro lado, a análise de relações de interdependência pode ser executada através das seguintes técnicas: análise de agrupamentos, análise de correspondência, análise fatorial e escalonamento multidimensional.

Uma vez que o objetivo desta pesquisa consistiu em identificar a estrutura e padrões de relações existentes entre os indicadores de desempenho utilizados por empresas localizadas na cidade de Serra talhada/PE, a abordagem multivariada que analisa relações de interdependência é a apropriada para assegurar consistência metodológica aos procedimentos executados. Para esta pesquisa foram consideradas três técnicas, a saber:

- Análise de agrupamentos;

- Escalonamento multidimensional;

- Análise fatorial.

Pohlmann (2007) considera que a análise de agrupamentos (ou conglomerados) se caracteriza por ser descritiva, ateorética e não inferencial, não tendo base estatística para formular inferências sobre uma população com base em uma amostra e sendo usada como técnica exploratória.

O escalonamento multidimensional é definido por Herdeiro (2007) como sendo uma técnica estatística que analisa posicionamentos, comparações de padrão, graus de proximidade e classificações por afinidade entre diversos elementos.

O escalonamento multidimensional é definido por Herdeiro (2007) como sendo uma técnica estatística que analisa posicionamentos, comparações de padrão, graus de proximidade e classificações por afinidade entre diversos elementos.

Bezerra (2007) define análise fatorial como sendo uma técnica estatística que busca identificar dimensões de variabilidade comuns existentes entre u conjunto de fenômenos. Sobre os procedimentos referentes à escolha da técnica de rotação de fatores adequada, Hair et al. (2005) afirmam que não há nenhuma regra específica para a definição da técnica a ser utilizada, mas informam que a técnica mais utilizada é a VARIMAX, por estar disponível na maioria dos aplicativos estatísticos computacionais.

Esta pesquisa considerou a utilização de mais de uma técnica multivariada em função da intenção de caracterizar a estrutura de relações e padrões de uso referentes aos indicadores de mensuração de desempenho no âmbito das empresas investigadas, bem como para comparar os resultados obtidos. 
Considerando a possibilidade de utilização de mais de uma técnica, Hair et al (2005) ressalta que as técnicas multivariadas que investigam interdependências podem ser comparadas entre si.

A operacionalização desta pesquisa considerou indicadores de mensuração de desempenho estudados como sendo binários, representando matematicamente o uso (1) e o não uso (zero). Todos os cálculos e demais procedimentos estatísticos foram efetuados a partir do aplicativo computacional STATISTICA for windows.

\section{APRESENTAÇÃO E ANÁLISE DOS RESULTADOS}

Como a análise dos resultados considerou uma perspectiva multivariada, faz-se necessário ressaltar que as técnicas utilizadas tiveram o objetivo de ilustrar a estrutura de relações inerentes ao uso dos diversos indicadores de mensuração de desempenho utilizados pelas empresas investigadas.

Este procedimento assume a premissa de que, na medida em que sejam encontradas proximidades entre alguns dos indicadores de desempenho, estes estejam relacionados entre si. A utilização de mais de uma técnica multivariada (análise de agrupamento, escalonamento multidimensional e análise fatorial) para analisar os resultados foi adotada para dar maior consistência e robustez aos resultados encontrados.

Para a execução da análise de agrupamento, foi primeiramente utilizado o procedimento de árvore de agrupamento. A regra de amalgamação adotada foi referenciada por elos simples e as distâncias foram calculadas a partir do procedimento city-block (manhattan). Os resultados estão dispostos no gráfico 1.

O gráfico gerado mostra os agrupamentos obtidos. Pode-se observar que, dos dezessete indicadores de desempenho investigados, apenas nove apresentaram-se relacionados de maneira significativa. Deste modo, os demais indicadores foram excluídos da pesquisa, pois não faria sentido considerá-los na análise dos resultados obtidos através do escalonamento multidimensional. A árvore de agrupamentos destaca a presença de três grupos significativos de variáveis. 


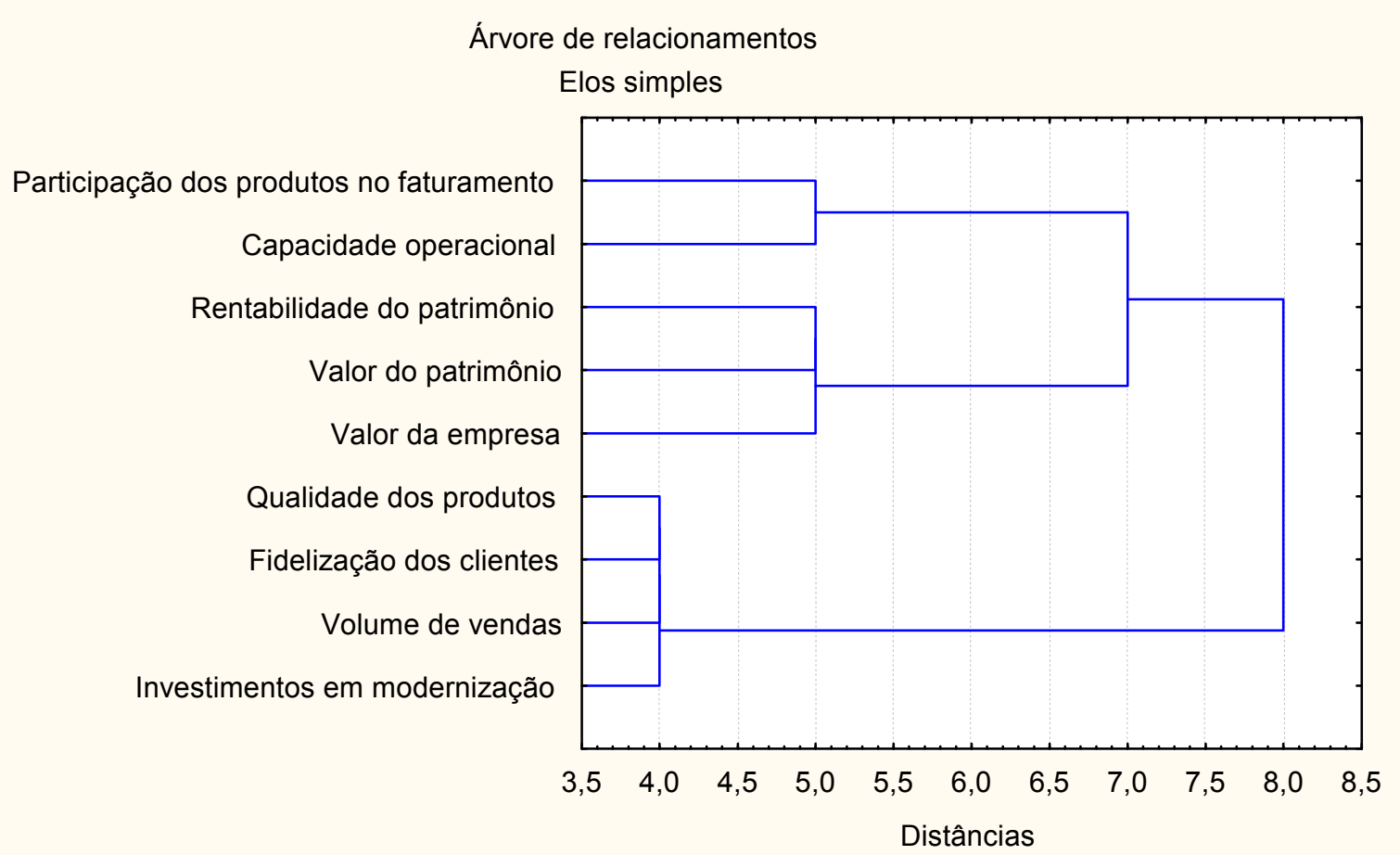

Gráfico 1 - Relação entre as variáveis através da análise de agrupamentos.

Fonte: Cálculos próprios.

Número de variáveis: 9

Número de casos: 46

Regra de Amalgamação: Elos simples

Mensuração das Distâncias: Distâncias City-block (Manhattan)

Dentre os distintos agrupamentos encontrados, um agrupamento apresenta relações internas muito significativas (que se dão no nível 4 da escala de distâncias).

Este grupo é composto pelos indicadores de desempenho que tratam da qualidade dos produtos, da fidelização dos clientes, do volume de vendas e dos investimentos em modernização. Este agrupamento se caracteriza por um perfil predominantemente qualitativo, no qual estão presentes vendas, ações de modernização, fidelização e qualidade. A configuração deste agrupamento traduz a relevância e a interdependência das dimensões representadas pelos indicadores de desempenho contidos em sua estrutura.

Ao identificar as relações significativas entre indicadores de desempenho, conforme é sugerido por Miranda e Silva (2002) como sendo desejáveis e relevantes, os achados obtidos sugerem que os atributos de desempenho mais relevantes a serem adotados como referência de avaliação devem ser identificados de maneira direta e objetiva.

O segundo agrupamento é formado por variáveis que representam a participação dos produtos no faturamento e pela capacidade operacional.

O terceiro agrupamento é composto pela rentabilidade, pelo valor do patrimônio e pelo valor da empresa. A intensidade das relações encontradas no núcleo destes dois últimos agrupamentos não foi tão significativa quanto às relações apresentadas no primeiro agrupamento (suas relações se deram no nível 4 da escala de distâncias).

Dando continuidade aos procedimentos de análise propostos para esta pesquisa, foi investigada a disposição multidimensional das relações entre os indicadores de desempenho considerados válidos pela análise de agrupamento para corroborar ou refutar os achados obtidos através da análise de agrupamentos. 
Em seguida, foi elaborado um gráfico bi-dimensional. Desta forma, a representação espacial da disposição das variáveis foi importante para identificar a disposição espacial das variáveis e verificar se há similaridade com a composição dos agrupamentos obtidos. Os resultados estão dispostos no gráfico 2 .

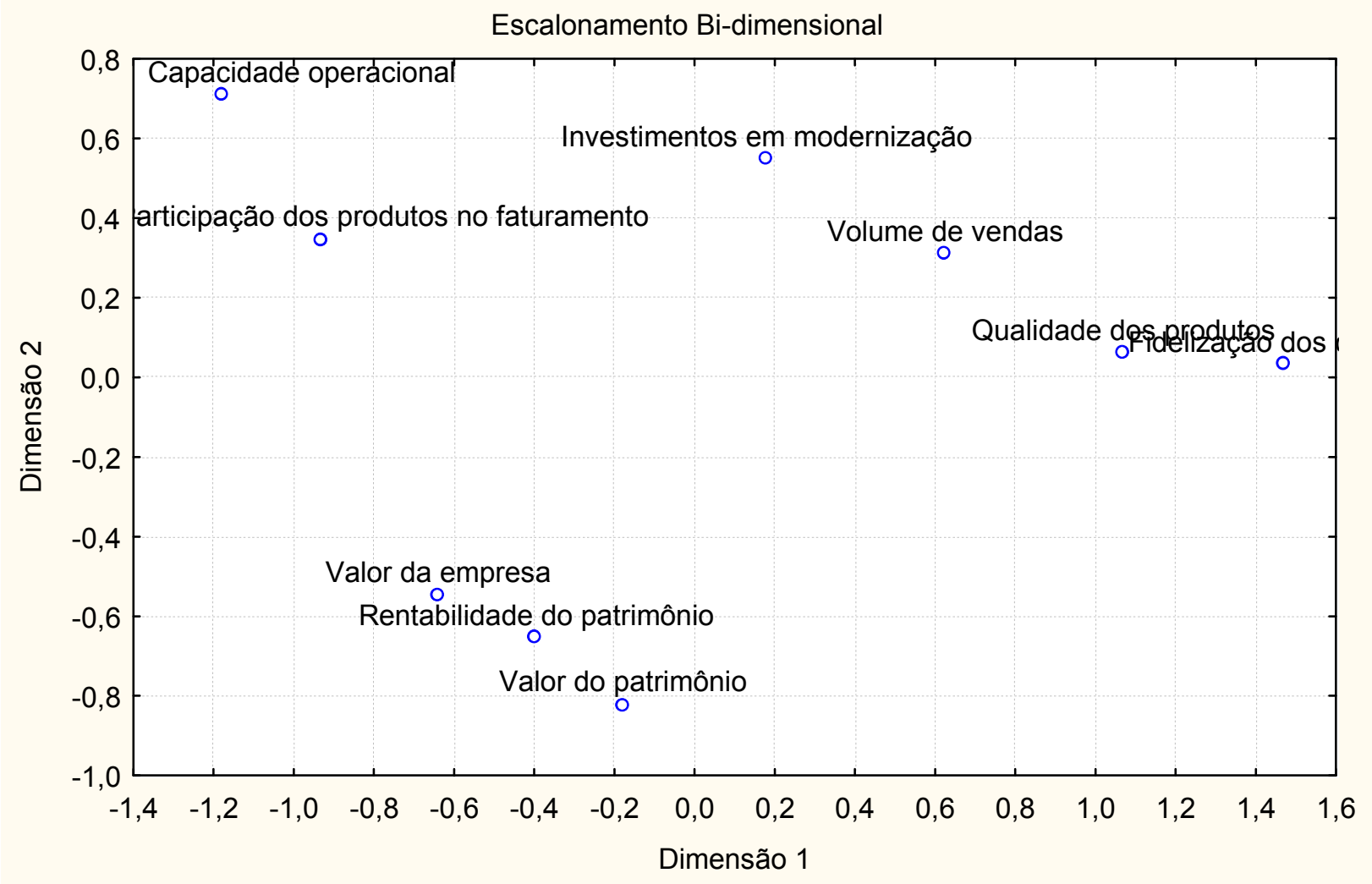

Gráfico 2 - Agrupamento das variáveis através do escalonamento bi-dimensional.

Fonte: Cálculos próprios.

Número de variáveis: 9

Número de dimensões: 2

Última interação computada: 80; Melhor iteração: 54

Alienação: 0,0662137

Estresse: 0,0381175

Os resultados apresentados através do gráfico bi-dimensional obtido corroboram a distribuição espacial dos indicadores de mensuração de desempenho dentro de cada um dos agrupamentos encontrados anteriormente, bem como confirma o relativo distanciamento entre eles.

Cada um dos indicadores de mensuração de desempenho pode ser visualizado individualmente através da distribuição espacial gerada pelo escalonamento bi-dimensional. Estes resultados são estatisticamente significativos. Os níveis de alienação e estresse ficaram inferiores a 0,05 .

Merece ainda ser destacado que, através do gráfico bi-dimensional, observa-se dentro do agrupamento principal que a variável qualidade do produto está situada praticamente sobre a variável referente à fidelização dos clientes.

Com a finalidade de corroborar os resultados obtidos e dar maior precisão à representação gráfica do escalonamento multidimensional, os cálculos foram executados novamente para gerar um gráfico tri-dimensional. 
O escalonamento tri-dimensional apresenta com maior nitidez a proximidade do relacionamento entre os diversos indicadores de desempenho inseridos em cada um dos agrupamentos significativos. Estes resultados confirmam os resultados obtidos através da análise de agrupamento.

\author{
Scatterplot 3D \\ Final Configuration
}

Dimension 1 vs. Dimension 2 vs. Dimension 3

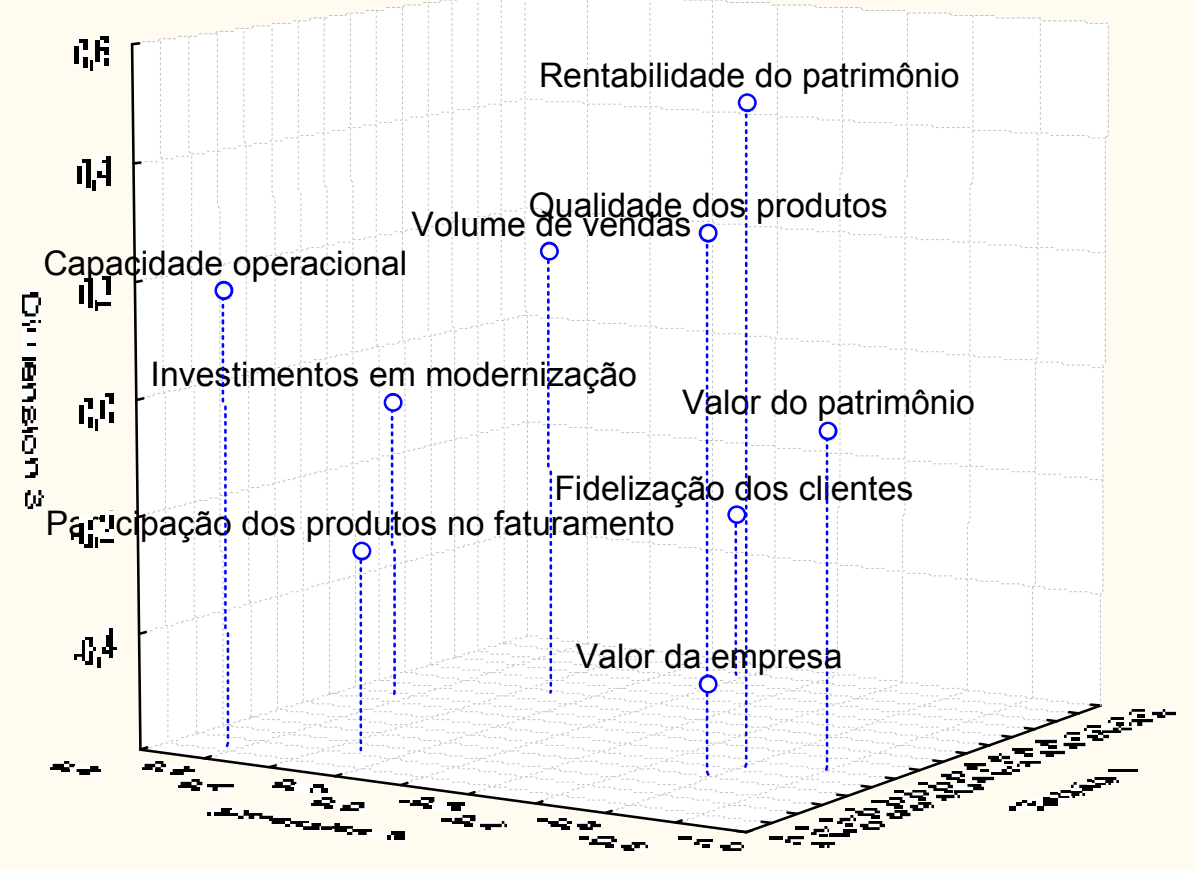

Gráfico 3 - Agrupamento das variáveis através do escalonamento tri-dimensional.

Fonte: Cálculos próprios.

Número de variáveis: 9

Número de dimensões: 3

Última interação computada: 146; Melhor iteração: 61.

Alienação: 0,0084429

Estresse: 0,0046542

E por último foi executada a análise fatorial. A configuração do cálculo ainda considerou a busca por três fatores, uma vez que as duas técnicas multivariadas anteriores evidenciaram a presença de três agrupamentos distintos, exigindo um mínimo eigenvalue equivalente a 1. Os resultados da análise fatorial não rotacionada estão apresentados na tabela 1.

Pode-se observar que foram identificados apenas dois fatores. O primeiro fator é composto pelos indicadores de mensuração de desempenho referentes à participação dos produtos no faturamento, à rentabilidade do patrimônio, ao valor da empresa, ao valor do patrimônio e à capacidade operacional. O segundo fator é composto pelos indicadores de mensuração de desempenho que representam a qualidade dos produtos, a fidelização dos clientes e o volume de vendas.

Os resultados obtidos através da análise fatorial demonstram que os três agrupamentos de indicadores de mensuração de desempenho encontrados podem ser considerados como 
pertencentes a duas categorias distintas. O primeiro fator contém os indicadores que representam a participação dos produtos no faturamento, a capacidade operacional, a rentabilidade, o valor do patrimônio e o valor da empresa.

O segundo fator significativo encontrado é composto pelos indicadores de desempenho pertencentes ao grupo de desempenho que apresentou as relações mais significativas (composto por qualidade dos produtos, da fidelização dos clientes, do volume de vendas) (excetuando a e dos investimentos em modernização). Este resultado indica que ambos sejam complementares entre si.

\begin{tabular}{ccc}
\hline Indicadores & Fator 1 & Fator 2 \\
\hline Participação dos produtos no faturamento & $-0,843068$ & 0,316716 \\
Rentabilidade do patrimônio & $-0,848437$ & 0,006533 \\
Valor da empresa & $-0,882615$ & 0,294959 \\
Valor do patrimônio & $-0,836119$ & $-0,005897$ \\
Qualidade dos produtos & $-0,072650$ & $-0,974644$ \\
Fidelização dos clientes & $-0,063444$ & $-0,919323$ \\
Volume de vendas & $-0,269759$ & $-0,924236$ \\
Capacidade operacional & $-0,761056$ & 0,231225 \\
Investimentos em modernização & $-0,507644$ & $-0,641157$ \\
\hline Capacidade explicativa & 0,425299 & 0,366804 \\
\hline
\end{tabular}

\section{Tabela 1 - Resultados da análise fatorial não-rotacionada}

Fonte: Cálculos próprios.

Método: Não-rotacionado

Número de variáveis: 9

Método: Componentes principais

Log (10) Determinante da matriz de correlação: -6,2526

Número de fatores extraídos: 2

Eigenvalues: Fator 1: 3,82769

Fator 2: 3,30123

Para dar mais consistência à análise fatorial realizada, o procedimento foi repetido considerado a rotação dos fatores. O método rotacional utilizado foi o VARIMAX. Os resultados estão dispostos na tabela 2.

Pode-se observar que, novamente, foram identificados apenas dois fatores distintos. O primeiro fator é composto pelos mesmos indicadores de mensuração de desempenho obtidos na primeira análise fatorial realizada. $O$ segundo fator obtido através da rotação também foi composto pelos mesmos indicadores de mensuração de desempenho, embora desta vez os investimentos em modernização tenham sido incorporados.

\begin{tabular}{ccc}
\hline Indicadores & Fator 1 & Fator 2 \\
\hline Participação dos produtos no faturamento & 0,890020 & $-0,137610$ \\
Rentabilidade do patrimônio & 0,831841 & 0,167116 \\
Valor da empresa & 0,924282 & $-0,108225$ \\
Valor do patrimônio & 0,817242 & 0,176764 \\
Qualidade dos produtos & $-0,128206$ & 0,968903 \\
Fidelização dos clientes & $-0,125904$ & 0,912868
\end{tabular}




\begin{tabular}{ccc}
\hline Volume de vendas & 0,075046 & 0,959870 \\
Capacidade operacional & 0,792258 & $-0,070697$ \\
Investimentos em modernização & 0,365795 & 0,731423 \\
\hline Capacidade explicativa & 0,422853 & 0,369250 \\
\hline
\end{tabular}

\section{Tabela 2 - Resultados da análise fatorial rotacionada}

Fonte: Cálculos próprios.

Método: Rotacionado

Número de variáveis: 9

Método: Componentes principais

Log (10) Determinante da matriz de correlação: $-6,2526$

Número de fatores extraídos: 2 Eigenvalues: Fator 1: 3,82769 Fator 2: 3,30123

Merece ainda ser destacado que apesar de cargas fatoriais de alguns dos indicadores de desempenho considerados na análise terem se alterados levemente, as estruturas dos fatores não se modificaram de maneira significativa.

\section{CONCLUSÕES}

O objetivo deste trabalho foi identificar a estrutura e padrões de relações existentes entre os indicadores de desempenho utilizados em empresas localizadas em Serra Talhada/PE. Para atingir o objetivo proposto, foi utilizada uma abordagem metodológica multivariada composta pela utilização de três técnicas distintas (análise de agrupamento, escalonamento multidimensional e análise fatorial).

Estas técnicas foram consideradas dentro de uma perspectiva comparativa na qual os resultados iguais obtidos teriam maior robustez ao ilustrar as relações entre os trinta e sete indicadores de mensuração de desempenho analisados. Os resultados obtidos demonstraram que apenas nove indicadores de desempenho puderam ser considerados como pertencentes a algum agrupamento de maneira significativa.

Através da árvore de agrupamentos foi observada a presença de três agrupamentos significativos de indicadores de mensuração de desempenho. Resultados semelhantes foram obtidos pelo escalonamento multidimensional. Em relação à análise fatorial, foram identificados dois fatores distintos que abrangem oito dos nove indicadores de desempenho que obtiveram relações estatisticamente significativas.

A dimensão de qualidade assume uma importância individual significativa, uma vez que as empresas investigadas apontaram estreitos vínculos entre a qualidade dos produtos, a fidelização dos clientes, o volume de vendas e os investimentos em modernização A dimensão de qualidade caracteriza o foco gerencial tradicional e apresenta uma visão interna para a mensuração do desempenho.

A dimensão econômica e a dimensão operacional fornecem uma visão externa ao atribuir uma importância gerencial para a participação dos produtos no faturamento, para a capacidade operacional, para a rentabilidade, para o valor do patrimônio e para o valor da empresa.

Estes resultados revelam indícios particulares referentes à caracterização da dinâmica das relações entre os principais indicadores de desempenho utilizados pelas empresas localizadas em Serra Talhada/PE.

Conforme foi apresentado pela literatura, a análise do desempenho pressupõe uma perspectiva múltipla e complementar de diversas perspectivas. Os resultados obtidos corroboram este postulado teórico e dão sustentação à necessidade gerencial de construir um 
elenco de indicadores de desempenho que seja diversificado, mas que considere os aspectos mais relevantes para o setor no qual a empresa atue.

Apesar dos resultados obtidos serem estatisticamente significativos, não há sustentação suficiente para se afirmar categoricamente que os três grupos encontrados sejam independentes entre si ou que eles não exerçam contribuições individuais complementares para com o desempenho das empresas do setor de hotelaria, uma vez que não foram encontrados três fatores distintos (o que corroboraria categoricamente a independência de cada um dos agrupamentos de indicadores em relação aos demais).

Estudos mais aprofundados poderão gerar informações mais detalhadas sobre a natureza das relações dinâmicas encontradas nesta pesquisa e contribuir para o aprimoramento da base de conhecimento existente sobre as relações dinâmicas existentes no âmbito das práticas gerenciais voltadas para o uso de indicadores de desempenho.

\section{REFERENNCIAS}

ANDRADE, M. M. Introdução à metodologia do trabalho científico. 4. ed. São Paulo: Atlas, 1999.

ARAÚJO, A. O. Contribuição ao estudo de indicadores de desempenho de empreendimentos hoteleiros, sob o enfoque da gestão estratégica. Tese de Doutorado em Ciências Contábeis, São Paulo, Universidade de São Paulo, 2001.

BANKER, R. D; POTTER, G; SRINIVASAN, D. An empirical investigation of an incentive plan that includes nonfinancial performance measures. The Accounting Review, Florida, vol.75, n.1, p.65-92, jan, 2000.

BEZERRA, F. A. Análise fatorial. In: CORRAR, Luiz João; PAULO, Edílson; DIAS FILHO, José Maria (Org.). Análise Multivariada. São Paulo: Atlas, 2007.

BRIGNALL, S. A contingent rationale for cost system design in services. Management Accounting Research, vol. 8, n.3, p.325-346, 1997.

CHIZZOTTI, A. A Pesquisa em Ciências Humanas e Sociais. São Paulo: Cortez, 1991.

GIL, A. C. Métodos e Técnicas de Pesquisa Social. 5. ed. São Paulo: Atlas, 1999.

Como elaborar projetos de pesquisa. 4. ed. São Paulo: Atlas, 2002.

GONÇALVES, J. P. Desempenho Organizacional. Seminário Econômico. São Paulo, n. 815, ago/2002.

HAIR, J. F; ANDERSON, R. E; TATHAM, R. L; BLACK, W. C. Análise Multivariada de Dados. Porto Alegre: Bookman, 2005.

HERDEIRO, R. F. C. Escalonamento multidimensional. In: CORRAR, Luiz João; PAULO, Edílson; DIAS FILHO, José Maria (Org.). Análise Multivariada. São Paulo: Atlas, 2007. 
KAPLAN, R. S.; NORTON, D. P. The Balanced Scorecard: measures that drive performance. Harvard Business Review, Boston, jan-fev, p. 71-79, 1992.

KAPLAN, R. S; NORTON, D. P. A estratégia em ação: Balanced Scorecard. 9. ed. Rio de Janeiro: Campus, 1997. 344p.

LEITE, J. A. A. Metodologia de Elaboração de Teses. São Paulo: Editora McGraw-Hill do Brasil, 1978. 120p.

LEVIN, J. Estatística aplicada a ciências humanas. 2.ed. São Paulo: Harbra, 1987. 392p.

MACARTHUR, J. B. Performance measures that count: monitoring variables of strategic importance. Journal of Cost Management, v. 10, n. 3, p. 39-45, 1996.

MACEDO, M. A. S; SILVA, F. F. Análise de desempenho organizacional: utilizando indicadores financeiros e não financeiros na avaliação de performance empresarial. In: ENCONTRO DA ASSOCIAÇÃO NACIONAL DOS PROGRAMAS DE PÓSGRADUAÇÃO EM ADMINISTRAÇÃO, 28, 2004, Curitiba. Anais... Curitiba: ANPAD, 2004.

MARTINS, D. M Eficácia dos indicadores de desempenho na cadeia de fornecimento automotivo. In SIMPÓSIO DE ADMINISTRAÇÃO DA PRODUÇÃO, LOGÍSTICA E OPERAÇÕES INTERNACIONAIS, 7, 2004, São Paulo. Anais... São Paulo: SIMPOI, 2004.

MIRANDA. L. C. e SILVA, J. D. G. Medição de Desempenho. In: SCHMIDT, Paulo (Org.). Controladoria: Agregando Valor para a Empresa. Porto Alegre: Bookman, 2002.

MORISSETE, R. Toward a theory of information choices in organizations: an integrative approach. Ph.D. Dissertation in Accounting. University of Waterloo, 1977.

NAJMI, M; RIGAS, J; FAN, I. A framework to review performance measurement systems. Business Process Management Journal, Vol. 11. n 2., 2005.

NEELY, A; GREGORY, M; PLATTS, K. Performance Measurement System Design: should Process Based Approaches be adopted? International Journal Production Economics, Amsterdam, v. 46-47, 1996.

Performance Measurement System Design: A

literature review and research agenda. International Journal of Operations \& Production Management. v. 25. n .12, 2005.

POHLMANN, M. C. Análise de Conglomerados. In: CORRAR, Luiz João; PAULO, Edílson; DIAS FILHO, José Maria (Org.). Análise Multivariada. São Paulo: Atlas, 2007. 
RICHARDSON, R. J. Pesquisa social: métodos e técnicas. São Paulo: Atlas, 1999.

RODRIGUES, A.; PAULO, E. Introdução à análise multivariada. In: CORRAR, Luiz João; PAULO, Edílson; DIAS FILHO, José Maria (Org.). Análise Multivariada. São Paulo: Atlas, 2007.

SINK, S.; TUTLLE, T. C. Planning and measurement in your organization of the future. Norcross, Georgia: Industrial of Industrial Earnings, 1989.

SILVER, M. Estatística para Administração. São Paulo: Atlas, 2000.

VERGARA, S. C. Projetos e relatórios de pesquisa em administração. 4. ed. São Paulo: Atlas, 2003.

WALTER, F; BORNIA, A. C; KLIEMANN NETO, F. J. Análise comparativa de duas metodologias para elaboração do Balanced Scorecard. In: ENCONTRO DA ASSOCIAÇÃO NACIONAL DOS PROGRAMAS DE PÓS-GRADUAÇÃO EM ADMINISTRAÇÃO, 24, 2000, Florianópolis. Anais... Florianópolis: ANPAD, 2000

WOUTERS, M; SPORTEL, M. The role of existing measures in developing and implementing performance measurement systems. International Journal of Operations \& Production Management, Vol. 25. n .11, 2005.

ZILBER, M. A.; FISCHMANN, A. A. Competitividade e a importância de indicadores de desempenho: utilização de um modelo de tendência. In: ENCONTRO DA ASSOCIAÇÃO NACIONAL DOS PROGRAMAS DE PÓS-GRADUAÇÃO EM ADMINISTRAÇÃO, 26, 2002, Salvador. Anais... Salvador: ANPAD, 2002. 